\title{
Aktualisierte Anforderungen an die Messqualität und Qualitätssicherung (QS) von Point-of-Care-Testing(POCT)-Blutglukose-Messsystemen mit Unit- use-Reagenzien, die für die Erstdiagnostik eines manifesten Diabetes in der Schwangerschaft oder eines Gestationsdiabetes mellitus (GDM) gemäß der GDM-Leitlinie der Deutschen Diabetes Gesellschaft (DDG) geeignet sind
}

\author{
Konsensus-Empfehlung der Deutschen Vereinten Gesellschaft für Klinische \\ Chemie und Laboratoriumsmedizin (DGKL) mit der Deutschen Diabetes \\ Gesellschaft (DDG) 2015
}

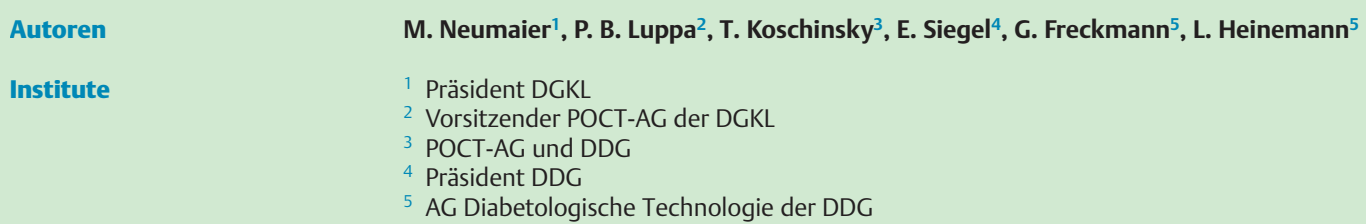

English version of the statement is available at http://dx.doi.org/ 10.1055/s-0035-1553622

\section{Bibliografie}

Dol http://dx.doi.org/ 10.1055/s-0035-1553518 Diabetologie 2015; 10: 197-199 @ Georg Thieme Verlag KG Stuttgart · New York . ISSN 1861-9002

Korrespondenzadresse Prof. Dr. T. Koschinsky POCT-AG und DDG München

tkoschinsky@t-online.de

\section{Grundlagen und Voraussetzungen $\nabla$}

Die DDG und die Deutsche Gesellschaft für Gynäkologie und Geburtshilfe (DGGG) haben im Jahr 2011 für den Gestationsdiabetes mellitus (GDM, ICD-10: 024.4G) eine neue, inter-disziplinäre, fachgesellschaftsübergreifende, evidenzbasierte S3-Leitlinie (AWMF-Leitlinie 057/008) veröffentlicht [1]. In dieser geben sie Empfehlungen zur Diagnostik, Therapie und Nachsorge des GDM. Durch diese Leitlinie wird erstmals auch der Einsatz von POCT-Glukose-Messsystemen mit Unit-use-Reagenzien bei der Erstdiagnostik des GDMdurch den betreuenden Arzt ermöglicht. Gleichzeitig wird eine entsprechende Herstellerempfehlung für diesen Einsatz zur Voraussetzung gemacht [1,2]: „Zur Erstdiagnostik eines manifesten Diabetes in der Schwangerschaft oder eines Gestationsdiabetes sollen bei Anwendung von Unit-use-Reagenzien und dazugehörigen Messsystemen diese - wie auch alle anderen Glukose-Messsysteme - nach Herstellerempfehlungen (Gebrauchsanweisung) für die ärztliche Anwendung in der Diagnostik ausdrücklich vorgesehen sein."

Gemeinsam ist dieser Leitlinie wie den anderen diesbezüglichen DDG-Leitlinien [1 -3], dass Handmessgeräte zur Blutglukose(BG)-Selbstmessung (Homecare-Bereich) für die Primärdiagnostik eines GDM wie auch aller anderen Diabetesformen ungeeignet sind. D.h., die Verwendung von „Patientenmesssystemen“ ist gemäß allen diesbezüglichen Leitlinien abzulehnen, da diese Systeme bei ihrer europäischen Zulassung mit einer CE-Kennzeichnung nur die Minimalanforderung nach dem entsprechenden ISO-Standard 15197 erfüllen müssen, d. h., die Güte der Glukosemessung im klinisch-relevanten Messbereich darf für $95 \%$ aller Werte noch bis Mai 2016 bis $\mathrm{zu} \pm 20 \%$ vom Referenzwert abweichen und erst ab Juni 2016 nicht mehr als $\pm 15 \%$ - ein für pri- märdiagnostische Messungen nicht akzeptabler maximal zulässiger Messfehler.

Die Aktualität und die praktische Relevanz der neuen GDM-Leitlinie gehen auch daraus hervor, dass ihre wesentlichen Inhalte auch vom Gemeinsamen Bundesausschuss in seiner Sitzung am 15.12.2011 in den Beschluss übernommen wurden, die Richtlinien über die ärztliche Betreuung während der Schwangerschaft und nach der Entbindung (Mutterschafts-Richtlinien) entsprechend zu ergänzen [4].

Da die o. g. DDG-Leitlinien [1 - 3] keine detaillierten Vorgaben für die geforderten Herstellerempfehlungen enthielten, wurden im Jahr 2012 die Anforderungen an die Messqualität und die Qualitätssicherung von POCT-Glukose-Messsystemen für die primäre GDM-Diagnostik durch eine gemeinsame Stellungnahme der Deutschen Vereinten Gesellschaft für Klinische Chemie und Laboratoriumsmedizin (DGKL) und der DDG unter Berücksichtigung der zu diesem Zeitpunkt verfügbaren technischen Möglichkeiten konkretisiert [5].

Da seither die Messgüte bei den besten verfügbaren POCT-Glukose-Messsysteme mit Unit-useReagenzien weiter wesentlich verbessert wurde [6-11], besteht die Möglichkeit (und wir sehen den Bedarf), die bisherigen Anforderungen insbesondere an deren Messqualität beim Einsatz für die Diagnostik eines manifesten Diabetes in der Schwangerschaft oder eines GDM durch DGKL und DDG gemeinsam zu aktualisieren ( $\bullet$ Tab. 1). Damit soll den Herstellern, den Zulassungs- und Regulierungsbehörden, den Nutzern bei klinischen Studien und im Praxisalltag sowie den Kostenträgern eine evidenzbasierte Orientierung gegeben werden. 
Tab. 1 Vergleich der Mindestanforderungen an die Messqualität und die QS von POCT-Glukose-Messsystemen mit Unit-use-Reagenzien für die GDM-Diagnostik gemäß den Empfehlungen der DGKL und DDG von 2012 und 2015.

\begin{tabular}{|c|c|c|}
\hline & 2012 & 2015 \\
\hline Zulassungskriterien & Conformité Européene: CE-Zeichen & Conformité Européene: CE-Zeichen \\
\hline Untersuchungsprobe & venöses Vollblut (kein Kapillarblut) & venöses Vollblut (kein Kapillarblut) \\
\hline $\begin{array}{l}\text { System-Richtigkeit (Accuracy) gemäß Prüf- } \\
\text { standard mit folgenden Modifikationen: }\end{array}$ & DIN EN ISO 15 197:2003 & DIN EN ISO 15 197:2013 \\
\hline Blutglukose-Konzentration & $<75 \mathrm{mg} / \mathrm{dL} \quad>75 \mathrm{mg} / \mathrm{dL}$ & $<100 \mathrm{mg} / \mathrm{dL} \quad>100 \mathrm{mg} / \mathrm{dL}$ \\
\hline Toleranzbereich & $\pm 10 \mathrm{mg} / \mathrm{dL} \quad \pm 15 \%$ & $\pm 10 \mathrm{mg} / \mathrm{dL} \quad \pm 10 \%$ \\
\hline Mindestanforderung & $95 \%$ aller Messwerte im Toleranzbereich & $95 \%$ aller Messwerte im Toleranzbereich \\
\hline $\begin{array}{l}\text { Sicherstellung (herstellerseitig) dieser } \\
\text { Modifikationen auch nach Zulassung }\end{array}$ & $\begin{array}{l}\text { für jede neue Charge von Teststreifen mit pro- } \\
\text { duktspezifischen Prüfverfahren vom Hersteller }\end{array}$ & $\begin{array}{l}\text { für jede neue Charge von Teststreifen mit pro- } \\
\text { duktspezifischen Prüfverfahren vom Hersteller; } \\
\text { bei inakzeptabler externer QS besteht Nachweis- } \\
\text { pflicht des Herstellers über das Ergebnis seines } \\
\text { diesbezüglichen Prüfverfahrens auf Anforderung } \\
\text { durch die Regulierungsbehörde. }\end{array}$ \\
\hline $\begin{array}{l}\text { Sicherung der Messqualität nach CE } \\
\text { interne Qualitätssicherung (QS) } \\
\text { externe QS (modifiziert) }\end{array}$ & $\begin{array}{l}\text { RiliBÄK } 2008 \\
1 \times / \text { Woche } \\
\text { verbindliche Teilnahme auch im Bereich des } \\
\text { niedergelassenen Arztes }\end{array}$ & $\begin{array}{l}\text { RiliBÄK } 2014 \\
\text { modifiziert: an allen Messtagen } \\
\text { verbindliche Teilnahme auch im Bereich des } \\
\text { niedergelassenen Arztes }\end{array}$ \\
\hline
\end{tabular}

\section{Aktualisierung der Anforderungen an die Messqualität und die Qualitätssicherung von POCT-Glukose-Mess- systemen mit Unit-use-Reagenzien für die Diagnostik eines manifesten Diabetes in der Schwangerschaft oder eines GDM (2015) \\ $\nabla$}

1. Zulassungskriterien für das CE-Zeichen: wie bisher, mit Fokus auf die Diagnostik eines manifesten Diabetes in der Schwangerschaft oder eines GDM.

2. Untersuchungsprobe: nur venöses Vollblut, kein Kapillarblut!

3. Nachweis der Richtigkeit (Accuracy) im gesamten klinisch relevanten Messbereich:

3.1 Bei der Zulassung von Messgerät und Teststreifen/Sensor in Deutschland/EU gemäß DIN EN ISO 15 197:2013 mit folgender Modifikation: mindestens 95\% der BG-Einzelergebnisse sollen innerhalb von $\pm 10 \mathrm{mg} / \mathrm{dL}$ im Vergleich zu den Messwerten mit dem Referenzsystem (Hexokinasemethodik oder GOD-Methodik mit YSI-System) des Herstellers bei BG-Konzentrationen $<100 \mathrm{mg} / \mathrm{dL}$ und innerhalb von $\pm 10 \%$ im Vergleich zu dem o. g. Referenzsystem bei BG-Konzentrationen $\geq 100 \mathrm{mg} / \mathrm{dL}$ liegen.

3.2 Bei der Produktion neuer Chargen von Teststreifen/Sensoren nach deren Zulassung: Herstellerseitig muss sichergestellt werden, dass jede neue Charge, die auf den Markt kommt, die Vorgaben gemäß 3.1 einhält. Das dafür geeignete Prüfverfahren kann vom Hersteller produktspezifisch festgelegt werden. Wenn die Messqualität neuer Chargen während des Gebrauchs nicht gemäß 4. gesichert werden kann, besteht eine Nachweispflicht des Herstellers über das Ergebnis seines diesbezüglichen Prüfverfahrens gemäß 3. auf Anforderung durch die Regulierungsbehörde. 4. Sicherung der Messqualität während des Gebrauchs:

4.1 Regelungen der RiliBÄK [12]: Alle diesbezüglichen Vorgaben der RiliBÄK für POCT-Systeme mit Unit-use-Reagenzien sind einzuhalten, einschließlich der Regeln für die interne und externe Qualitätssicherung (QS).

Die interne QS erfolgt mittels Kontrollprobeneinzelmessung. Als Kontrollen sind mindestens zwei unterschiedliche Glukosekonzentrationen einzusetzen, soweit eine entsprechende zweite Kontrolllösung verfügbar ist. Dafür beträgt die maximal zulässige Abweichung des Kontrollwerts vom Zielwert $11 \%$ im Gültigkeitsbereich von $40-400 \mathrm{mg} / \mathrm{dL}$. Beim Einsatz eines
POCT-Systems mit Unit-use-Reagenzien an mehreren Messtagen/Woche erfolgt die interne QS an allen Messtagen statt einmal wöchentlich (Minimalanforderung der RiliBÄK).

Bei der externen QS beträgt die maximal zulässige Abweichung beim Ringversuch für jede Glukose-Messmethodik im Labor wie im POCT-Bereich $15 \%$.

Derzeit muss aber bei den o.g. POCT-Glukose-Messsystemen wegen des Fehlens geeigneter Vollblutkontrollproben immer noch auf die RiliBÄK-Sonderregelungen zur Verwendung von Kontrollproben mit methodenspezifischen Zielwerten statt des Referenzmethodenwerts zurückgegriffen werden, woraus keine Aussagen zur Richtigkeit von POCT-Glukosemessgeräten abgeleitet werden können. Zu Recht wird daher gefordert, dass ,in dieser Situation Hersteller, Ringversuchsorganisationen und Kontrollmaterialhersteller aufgerufen sind, nach einem geeigneten Kontrollprobenmaterial zu suchen und dieses dann zur Verfügung zu stellen“ [13]. Bis zu diesem Zeitpunkt ist die Anwendung des sogenannten „Consensus Value“ ein praktikabler Lösungsansatz [13], wie er von den beiden Ringversuchsorganisationen (Referenzinstitut für Bioanalytik und INSTAND e.V.) zur vergleichenden Beurteilung erprobt worden ist.

4.2 Zusätzliche Regelung der GDM-Leitlinie der DDG und der DGGG [1, 2]: Im Bereich des niedergelassenen Arztes ist auch für POCT-Glukose-Messsysteme mit Unit-use-Reagenzien die Teilnahme an der externen QS analog zur RiliBÄK-Regelung verbindlich.

Interessenkonflikte: Der korrespondierende Autor, sowie die Koautoren hatten während der letzten drei Jahre keine wirtschaftlichen oder persönlichen Verbindungen in Bezug auf das Manuskript.

\section{Literatur}

1 Kleinwechter H, Schäfer-Graf U, Bührer C et al. Gestationsdiabetes mellitus (GDM). Evidenzbasierte Leitlinie zu Diagnostik, Therapie und Nachsorge der DDG und der DGGG. Diabetologie Stoffw 2011; 6: $290-328$

2 Kleinwechter H, Schäfer-Graf U, Bührer C et al. Gestationsdiabetes mellitus (GDM) - Diagnostik, Therapie und Nachsorge. Diabetologie Stoffw 2014; 9 (Suppl 2): S202-S213 in: Praxisempfehlungen der Deutschen Diabetes-Gesellschaft, Aktualisierte Version 2014, Kellerer 
M, Siegel E (Hrsg.) sowie im Internet: „Aktuelle Fassung der evidenzbasierten Leitlinien“ www.deutsche-diabetes-gesellschaft.de

3 Kerner W, Brückel J. Definition, Klassifikation und Diagnostik des Diabetes mellitus. Diabetologie Stoffw 2014; 9 (Suppl 2): S96-S99 in: Praxisempfehlungen der Deutschen Diabetes-Gesellschaft, Aktualisierte Version 2014, Kellerer M, Siegel E (Hrsg.) sowie im Internet: „Aktuelle Fassung der evidenzbasierten Leitlinien" www.deutsche- diabetes-gesellschaft.de

4 Beschluss des Gemeinsamen Bundesausschusses zum Screening auf Gestationsdiabetes mellitus im Internet: G-BA_Beschluss_2011-1215_Mu-RL_Screening_GDM.pdf

5 Thiery J, Luppa PB, Koschinsky T et al. Anforderungen an die Messqualität und Qualitätssicherung (QS) von Point-of-Care-Testing (POCT)Blutglukose-Messsystemen, die für das Screening und die Diagnose eines Gestationsdiabetes mellitus (GDM) gemäß der GDM-Leitlinie der Deutschen Diabetes-Gesellschaft (DDG) geeignet sind: Eine Initiative der POCT-AG der Deutschen Vereinten Gesellschaft für Klinische Chemie und Laboratoriumsmedizin in Abstimmung mit der DDG Dezember 2012. Diabetologie Stoffw 2013; 8: 195-197

6 Freckmann G, Baumstark A, Schmid C et al. Evaluation of 12 blood glucose monitoring systems for self-testing: System accuracy and measurement reproducibility. Diabetes Technol Ther 2014; 16: 113-122

7 Klaff LJ, Brazg R, Hughes K et al. Accuracy evaluation of Contour Next compared with five blood glucose monitoring systems across a wide range of blood glucose concentrations occurring in a clinical research setting. Diabetes Technol Ther 2014; 17: 8-15
8 Pleus S, Schmid C, Link M et al. Accuracy assessment of two novel systems for self-monitoring of blood glucose following ISO 15197:2013. J Diabetes Sci Technol 2014; 8: 906-908

9 Freckmann G, Schmid C, Pleus $S$ et al. System accuracy evaluation of systems for point-of-care testing of blood glucose: a comparison of a patient-use system with six professional-use systems. Clin Chem Lab Med 2014; 52: 1079-1086

10 Halldorsdottir S, Warchal-Windham ME, Wallace JF et al. Accuracy evaluation of five blood glucose monitoring systems: the North American comparator trial. J Diabetes Sci Technol 2013; 7: 1294-1304

11 Freckmann G, Pleus S, Link $M$ et al. Accuracy evaluation of four blood glucose monitoring systems in unaltered blood samples in the low glycemic range and blood samples in the concentration range defined by ISO-15197. Diabetes Technol Ther 2015

12 Bundesärztekammer. Richtlinie der Bundesärztekammer zur Qualitätssicherung laboratoriumsmedizinischer Untersuchungen (RiliBÄK 2014). Dtsch Ärztebl 2014; 111: A1583 - A1618

13 Petersmann A, Luppa P, Michelsen A et al. Gemeinsame Stellungnahme zur Situation der Bewertung von Ringversuchen für Glukose mittels Systemen für die patientennahe Sofortdiagnostik (POCT). J Lab Med 2012; 36: $165-168$ 